\title{
Wirksamkeit pflanzlicher Arzneimittel
}

\author{
S. Chrubasik
}

Institut für Rechtsmedizin, Universität Freiburg

Die 1989 gegründete European Scientific Cooperative on Phytotherapy (ESCOP, www.escop.com/) ist die Dachorganisation der nationalen Phytotherapiegesellschaften in Europa. Ihr Ziel ist die Harmonisierung der Zulassungen für pflanzliche Arzneimittel in Europa. Die seit 1997 publizierten ESCOP-Monographien enthalten die wesentlichen Informationen zu derzeit 60 Arzneipflanzen: die therapeutischen Indikationen, die pharmakologische und klinische Wirksamkeit, die Art der Applikation und die tägliche Dosierung sowie wesentliche Informationen, die bei der Anwendung beachtet werden müssen (Nebenwirkungen, Kontraindikationen usw.). Die Ergebnisse der klinischen Studien werden in den Monographien nur deskriptiv dargestellt, ohne dass die Qualität der Studien berücksichtigt wird. Wie evident die klinische Wirksamkeit ist, bleibt deshalb offen.

Die im deutschen Arzneimittelgesetz implementierten Empfehlungen der International Conference on Harmonization (ICH) for Good Clinical Practice (GCP) (www.ifpma.org/ ich5.html) enthalten Richtlinien zur Durchführung klinischer Studien (http://www.ich.org/pdfICH/e8.pdf). Sie enthalten Qualitätskriterien, die bei der Durchführung klinischer Studien Berücksichtigung finden sollten. Studien zur Wirksamkeit einer Medikation sollten randomisiert und doppelblind durchgeführt werden, Ein- und Ausschlusskriterien sowie die Zielkriterien sollten explizit genannt sein, die verwendeten Messinstrumente validiert sein. Zum Hauptzielkriterium sollten eine Nullhypothese und eine alternative Hypothese zwecks Fallzahlberechnung (die Power sollte möglichst nicht unter $80 \%$ liegen) vorliegen. Die charakteristischen Merkmale der Gruppen vor Beginn der Studie müssen umfassend dokumentiert sein, um Gruppenunterschiede und mögliche Prädiktoren auf das Ergebnis erkennen zu können. Ebenso müssen Kobehandlungen, Interventionen, Protokollverstösse und unerwünschte Wirkungen dokumentiert und die Methoden der statistischen Auswertung deklariert werden. In die Auswertung sollten alle Studienteilnehmer einbezogen werden («Intention-to-treat»-Analyse), unter Berücksichtigung potentieller Confounder und Interaktionen mit entsprechender Adjustierung. Auch Sensitivitätsanalysen tragen zur Transparenz der Robustheit der Ergebnisse bei, und Subgruppenanalysen können richtungsweisend sein.
Wenn (fast) alle Qualitätskriterien berücksichtigt werden, wird die interne Validität der Studie als «gut» bezeichnet [1]. «Moderate» Studien dürfen keine fatalen Schwächen besitzen, die das Ergebnis beeinflussen. «Schlechte» Studien sind nicht transparent, und das Ergebnis wird zum Beispiel durch Verwendung artefizieller Messinstrumente, inadäquater statistischer Vefahren oder Unausgeglichenheit der Studiengruppen usw. beeinflusst. Nur bei Studien mit guter interner Validität kann die Qualität der Evidenz beurteilt werden. Eine Studie ist konfirmativ (gute Evidenzqualität, beweisend), wenn (fast) alle Qualitätskriterien berücksichtigt wurden. Ansonsten muss die Studie als explorativ gewertet werden und besitzt entweder eine moderate (Wirksamkeitstrend) oder schlechte Evidenz (Wirksamkeit nicht belegt) [1]. Die Evidenzqualität sagt nichts über das Ausmass der Wirksamkeit aus. Der Nutzen und die potentielle Schädigung können an einer 4-PunkteSkala eingestuft werden: beachtlich, mässig, gering, null/negativ. Als beachtlicher Nutzen wird bereits eine kleine Besserung bei einem weitverbreiteten Beschwerdebild angesehen. Der Netto-Nutzen ergibt sich aus dem Abwägen von Nutzen und potentieller Schädigung.

Eine gut durchgeführte offene kontrollierte Studie kann ein aussagekräftigeres Ergebnis haben als eine schlecht durchgeführte verblindete Studie mit geringer Power zur Fallzahl. Die hierarchische Einteilung mit Erteilung der höchsten Punktzahl für randomisierte kontrollierte Studien wurde deshalb abgelöst durch eine Einstufung, die die Qualität klinischer Studien berücksichtigt [1]. Leider ist die interne Validität der meisten Studien mit pflanzlichen Arzneimitteln schlecht. Um die Wirksamkeit pflanzlicher Arzneimittel besser zu erfassen, werden deshalb zunehmend die Daten vieler Studien systematisch und methodisch nach vorgegebenen Kriterien erfasst. Wurde in verschiedenen Studien dasselbe Messinstrument zum selben Messzeitpunkt genutzt, kann aus gepoolten Daten der Nutzen des pflanzlichen Arzneimittels errechnet werden (siehe Cochrane-Reviews www.cochrane.de/cochrane/general. htm - kontinuierlich Aktualisierung - und www.ex.ac.uk/sshs/ compmed/publications/publications02.htm).

Für viele pflanzliche Arzneimittel ist der Wirksamkeitsnachweis noch nicht erbracht, nur zu einigen gibt es systematische

\begin{tabular}{ll}
\hline KARGER & $\oplus$ 2003 S. Karger GmbH, Freiburg \\
Fax +49761 4520714 & Accessible online at: \\
$\begin{array}{l}\text { E-mail Information@Karger.de } \\
\text { www.karger.com }\end{array}$ & www.karger.com/journals/fkm
\end{tabular}

Dr. Sigrun Chrubasik 
Reviews und Meta-Analysen. Für keines der pflanzlichen Arzneimittel gibt es eine verlässliche Aussage zum NutzenSchaden-Verhältnis, da Langzeitdokumentationen zur Wirksamkeit und Sicherheit fehlen. Auch fehlen vergleichende Untersuchungen zu den verschieden hergestellten Extrakten und Dosis-Wirkungs-Beziehungen, da die vorgeschlagenen Dosierungen fast alle auf Empirie beruhen. Die Durchführung von Wirksamkeitsstudien mit guter interner Validität ist dringend erforderlich, wenn die pflanzlichen Arzneimittel im Therapieschema einen festen Platz einnehmen sollen.

Zu den Gingko-biloba-Spezialextrakten EGb 761 und LI 1370 liegen einige Meta-Analysen vor, die Überlegenheit gegenüber Plazebo bei der Demenz vom Alzheimertyp, bei vaskulärer Demenz, altersbedingter Abnahme der Hirnleistung und Claudicatio intermittens demonstrieren. Nur wenige Daten liegen zum Vergleich von Gingko mit der konventionellen Therapie vor. Ob Gingko biloba bei der Behandlung von Tinnitus oder Makuladegeneration Wirksamkeit besitzt, lässt sich aufgrund der insuffizienten Datenlage derzeit noch nicht beurteilen [2]. Aus einer Cochrane-Meta-Analyse geht hervor, dass Hypericum-Präparate bei der Behandlung leichter bis mittelschwerer Depressionen einer Plazebobehandlung überlegen sind Ob Hypericum-Präparate ebenso wirksam sind wie synthetische Antidepressiva lässt sich zur Zeit noch nicht endgültig beurteilen. In seltenen Fällen können bei gleichzeitiger Gabe von Hypericum-Präparaten mit Herzglykosiden, Antikoagulanzien oder Immunsuppressiva durch Interaktionen beim Abbau der Medikamente schwerwiegende Komplikationen auftreten.

Ein systematisches Cochrane-Review mit Meta-Analyse demonstriert, dass Kava-Extrakt bei der Behandlung von Angstzuständen wirksamer ist als eine Plazebobehandlung. Aufgrund von Todesfällen durch medikamenteninduzierte Hepatitis (vor allem bei gleichzeitiger Einnahme lebertoxischer Medikamente oder Alkohol) wurden die Kava-Präparate in Deutschland aus dem Handel gezogen, obwohl die Richtigkeit dieser Entscheidung von den Experten der Kommission E angezweifelt wird.

Systematische Cochrane-Reviews zu Rosskastanien-Samenextrakt (Behandlung der chronischen venösen Insuffizienz), Serenoa repens, Pygeum africanum, Secale cereale (Cernilton ${ }^{\mathrm{R}}$ ) (Behandlung von Beschwerden bei benigner Prostatahyper- plasie Alken Stadium I bis II) und Echinacea-Präparaten (Behandlung oder Vorbeugung von Erkältungskrankheiten) lassen ebenfalls Wirksamkeiten erkennen. Die Evidenzlage zu Serenoa repens ist dabei sehr viel besser als zu Pygeum africanum und Secale cereale. Auch lässt sich nicht erkennen, welchem Echinacea-Präparat Wirksamkeit zugeschrieben werden kann. Eine Verlängerung der Gehstrecke bei arterieller Verschlusskrankheit durch Einnahme von Knoblauch konnte in einem systematischen Cochrane-Review dagegen nicht bestätigt werden. Zwei Meta-Analysen [3, 4] demonstrieren, dass bei erhöhten Cholesterinkonzentrationen im Serum die Behandlung mit Knoblauch einer Plazebobehandlung überlegen ist. Jedoch ist das Ausmass der Wirksamkeit gering (Reduktion unter $15 \%$ ) und nicht unumstritten. Nur bei geringer Erhöhung des Blutdrucks fand sich eine blutdrucksenkende Wirkung [5]. Die Hypothese, dass regelmässiger Genuss von Knoblauch von mehr als 4-29 g pro Woche eine krebsprophylaktische Wirkung besitzt, ist möglich, aber noch nicht eindeutig belegt [6].

Vielversprechend (doch noch nicht ausreichend belegt) ist die Datenlage zur cholesterinsenkenden Wirkung der Artischockenblätter, der migräneprophylaktischen Wirkung des Fieberkrauts und der blutzucker- und lipidsenkenden Wirkung von Aloe vera (Cochrane-Reviews). Zur Wirksamkeitsbeurteilung nicht ausreichend waren hingegen die Studien mit $\mathrm{Ci}$ micifuga racemosa (Behandlung klimakterischer Beschwerden), Baldrian (Behandlung von Schlafstörungen, [7]), Ginseng (Behandlung verschiedener Beschwerden) und Ingwer (Behandlung von Nausea und Erbrechen) (Cochrane-Reviews).

Die «ad hoc working group on herbal medicinal products» der European Agency for the Evaluation of Medicinal Products (EMEA) hat die Qualität pflanzlicher Drogen in einem umfassenden Dokument definiert, das bei künftigen Zulassungen in Europa grundlegend berücksichtigt werden muss (www. emea.eu.int/). Die Ergebnisse einer Studie mit einem speziellen Präparat können nicht auf andere Präparate übertragen werden, ohne den Nachweis, dass die Präparate bioäquivalent sind [8]. Denn wässrige Extrakte enthalten vor allem die wasserlöslichen Wirkbestandteile, während lipophile Extraktionsmittel die fettlöslichen Wirkbestandteile extrahieren. Mit Spezialverfahren können einzelne Wirkstoffe angereichert sein.

\section{Literatur}

1 Harris RP, Helfand M, Woolf S, Lohr KN, Mulrow CD, Teutsch SM, Atkins D: Current methods of the U.S. preventive service task force. Am J Prev Med 2001;20:21-35.

2 Duke RK, Chebib M, Duke CC, Johnston GAR, Roufogalis BD: Gingko biloba. Food for the brain. NZ Pharm 2002;22:27-33.

3 Silagy C, Neil A: Garlic as a lipid lowering agent - a meta-analysis. J R Coll Physicians Lond 1994;28: $39-45$.
4 Stevinson C, Pittler MH, Ernst E: Garlic for treating hypercholesterolaemia. A meta-analysis of randomised controlled studies. Ann Intern Med 2000;133:420-429.

5 Silagy CA, Neil HA: A meta-analysis of the effect of garlic on blood pressure. J Hypertens 1994;12: $463-468$.
6 Fleischauer AT, Poole C, Arab L: Garlic consumption and cancer prevention: Meta-analyses of colorectal and stomach cancers. Am J Clin Nutr 2000;72:1047-1052.

7 Stevinson C, Ernst E: Valerian for insomnia: A systematic review of randomized clinical trials. Sleep Med 2000;1:91-99.

8 Chrubasik S, Roufogalis B: Issues in quality and comparability of herbal medicinal products. Aust J Pharm 2001;82:444-445, 546-548. 